\title{
Ambenonium Chloride
}

National Cancer Institute

\section{Source}

National Cancer Institute. Ambenonium Chloride. NCI Thesaurus. Code C47388.

The chloride salt form of ambenonium, a quaternary ammonium compound with parasympathomimetic activity. Ambenonium chloride is a rapid indirect-acting cholinergic agonist that reversibly blocks the activity of acetylcholinesterase, thereby prevents acetylcholine hydrolysis and prolong ing its activity on nicotinic receptors at the neuromuscular junction. 